\title{
Bioinformatics analysis of key genes and latent pathway interactions based on the anaplastic thyroid carcinoma gene expression profile
}

\author{
YUN HUANG, YIMING TAO, XINYING LI, SHI CHANG, BO JIANG, FENG LI and ZHI-MING WANG \\ Department of Hepatobiliary Surgery, Xiangya Hospital, Central South University, Changsha, Hunan, P.R. China
}

Received July 16, 2015; Accepted November 10, 2016

DOI: $10.3892 / \mathrm{ol} .2016 .5447$

\begin{abstract}
Anaplastic thyroid carcinoma (ATC) is an aggressive malignant disease in older adults with a high mortality rate. The present study aimed to examine several key genes and pathways, which are associated with ATC. The GSE33630 gene expression profile was downloaded from the Gene Expression Omnibus database, which included 11 ATC and 45 normal thyroid samples. The differentially expressed genes (DEGs) in ATC were identified using the Limma package in $\mathrm{R}$. The Gene Ontology functions and Kyoto Encyclopedia of Genes and Genomes pathways of the selected DEGs were enriched using the Database for Annotation, Visualization and Integrated Discovery. A protein-protein interaction (PPI) network of the DEGs was constructed to select significant modules. Furthermore, a latent pathway interactive network was constructed to select the significant pathways associated with ATC. A total of 665 DEGs in the ATC samples were screened, and four significant modules were selected from the PPI network. The DEGs in the four modules were enriched in several functions and pathways. In addition, 29 significant pathways associated with ATC were selected, and he Toll-like receptor (TLR) signaling pathway, extracellular matrix (ECM)-receptor interaction and cytokine-cytokine interaction pathway were identified as important pathways. FBJ murine osteosarcoma viral oncogene homolog (FOS), chemokine C-X-C motif ligand 10 (CXCL10), collagen type $\mathrm{V} \alpha 1$ (COL5A1) and chemokine (C-C motif) ligand 28 (CCL28) were the key DEGs involved in these significant pathways. The data obtained in the present study revealed that the TLR signaling pathway, ECM-receptor interaction and cytokine-cytokine receptor interaction pathway, and the FOS, CXCL10, COL5A1, COL11A1 and CCL28 genes
\end{abstract}

Correspondence to: Dr Zhi-Ming Wang, Department of Hepatobiliary Surgery, Xiangya Hospital, Central South University, 87 Xiangya Road, Changsha, Hunan 410008, P.R. China

E-mail: zhimingwangzmw@163.com

Key words: anaplastic thyroid carcinoma, latent pathway analysis, differentially expressed gene, significant pathway, protein-protein interaction network have different roles in the progression of ATC, and these may be used as therapeutic targets for ATC.

\section{Introduction}

Thyroid cancer is one of the most common types of malignancy of the endocrine system (1). Anaplastic thyroid carcinoma (ATC), one of the four types of thyroid carcinoma, is an uncommon but aggressive malignancy in older adults, with a morbidity rate of $1.0-7.5 \%$, however, it accounts for $14-39 \%$ of thyroid carcinoma-associated mortality and the mean survival duration of ATC is usually $<6$ months from diagnosis (2). Although certain novel treatment methods, including surgery, genetic therapy and differentiation therapy, provide possibilities for the treatment of ATC (3), there is no effective systemic therapy for ATC. Therefore, it is important to examine the molecular mechanisms of ATC and identify several treatment methods for patients with a diagnosis of ATC.

The etiology of ATC remains to be fully elucidated. Previous studies have demonstrated that ionizing radiation, abnormal iodine intake, genetic factors and autoimmune disease are the primary factors contributing to the progression of ATC (4). Passaro et al (5) reported that ionizing radiation enhances the cell death of oncolytic adenovirus d1922-947 in ATC. Increased iodine intake contributes to a lower incidence of ATC (6), and it has been reported that forkhead box o3a enhances the proliferation of ATC cells via regulating the transcription factor, cyclin A1 (7). In addition, several pathways involved in the progression of ATC have been identified, including the Notch1 signaling pathway, phosphoinositide 3-kinase/Akt signaling pathway and the epidermal growth factor receptor/extracellular signal-regulated kinase pathway (8-10)

With developments in biology, several studies have focussed on to the mechanism of ATC. Consequently, several biomarkers have been identified for its treatment. For example, paired-box gene 8 may be a useful biomarker for ATC (11). In a study by Kim et al (12) L1 cell adhesion molecule was found to be overexpressed in patients with ATC and it may be an important therapeutic target for ATC treatment. In addition, the mutation of TP53 and expression of SRY-box 2 are associated with the progression from papillary thyroid carcinoma (PTC) to ATC (13). However, the mechanism underlying ATC remains to be fully elucidated. 
Microarray analysis is an effective approach to monitor global alterations of gene expression and identify the genes important to ATC. Several studies have been performed using the mRNA expression profile, GSE33630, of thyroid carcinoma. For example, Hébrant et al (14) found that ATC and PTC overlapped. He et al (15) analyzed 13 genes and one pathway associated with ATC. In addition, Xu et al found nine genes and one miscRNA, which were identified as candidates for the progression of thyroid cancer (16). However, the latent pathway interactions of ATC remain to be fully elucidated. Thus, using the same samples, the present study aimed to analyze the differentially expressed genes (DEGs) and significant pathways of ATC. Comprehensive bioinformatics analysis was used to enrich the significant functions and pathways of the DEGs to provide detailed insight into the biological mechanisms of ATC. This approach was used to predict the hub genes most likely associated with ATC. The present study aimed to provide a basis for further investigation of the mechanism of ATC.

\section{Materials and methods}

Data resources and data preprocessing. The GSE33630 gene expression profile data was downloaded from the Gene Expression Omnibus database (https://www.ncbi.nlm.nih.gov/geo/) from the National Center for Biotechnology Information based on the platform of the GPL570 Affymetrix Human Genome U133 Plus 2.0 array. The present study included a total of 105 samples, which comprised 11 ATC samples, 49 PTC samples and 45 normal thyroid (NT) samples. The 11 ATC samples and 45 NT samples were selected for analysis in the present study.

The CEL files were transformed into the expression value matrix using the Affy package in R (17), and the probe information was then transformed into the gene name using Bioconductor in $\mathrm{R}$ (18). If one gene had more than one probe, the mean expression value of this gene was selected.

DEG screening and enrichment analysis. The Limma package (19) in R language was used to select the DEGs in the ATC samples, compared with the NT samples. $\log 2$ fold-changel $>2$ and adjusted $\mathrm{P}<0.05$ were selected as the threshold values.

Gene Ontology (GO) analysis has become a commonly used approach for functional investigations of large-scale genomic or transcription data (20). The Kyoto Encyclopedia of Genes and Genomes (KEGG) pathway database contains information regarding how molecules or genes are networked, which provides complementary information to the majority of the existing molecular biology databases containing information on individual genes (21). The Database for Annotation, Visualization and Integrated Discovery (DAVID) bioinformatics resources consist of an integrated biological knowledge base and analytic tools aimed at systematically extracting biological meaning from large gene or protein lists (22). In the present study, the GO functions and pathways of the selected DEGs were enriched using DAVID in the GO (http://www.geneontology.org) and KEGG (http://www.genome.ad.jp/kegg/) databases with the adjusted $\mathrm{P}$-value (q-value) of $<0.05$.
PPI interaction network construction and selection of significant modules. The PPI interactive pairs of the screened DEGs were selected using the STRING online database (http://string-db.org/) (23). The DEG interactive pairs with a combined score of $>0.8$ were selected for the PPI network construction. Cytoscape (http://www.cytoscape.org/) was used to construct the PPI interaction network (24). The MCODE plugin was used to select the significant modules associated with ATC from the PPI interaction network (25). In addition, the GO terms and pathways of the DEGs in significant modules were enriched using DAVID (22) with $\mathrm{P}<0.05$.

Latent pathway identification analysis (LPIA). LPIA, developed by Pham et al (26), is a method for identifying the interactions of pathways associated with DEGs. A significant interaction represents a correlation between pathways and disease. The LPIA process was as follows: i) GO biological process (BP) terms (G) and KEGG pathways (P) of the DEGs were calculated using clusterProfiler (27) in R. ii) A bipartite network was constructed between $G$ and $P$, one edge of the node was $G$ and the other edge of node was $P$, with an edge representing one gene involved in $G$ and $P$, the weight of the edge was determined by the relative overlap of $\mathrm{G}$ and $\mathrm{P}$, which was calculated using Jaccard similarity coefficient and the mean expression value for the expression of each DEG. The formula to determine the weight was as follows:

$$
w_{G P}=\left|\frac{G \cap P}{G \cup P}\right| \times \operatorname{med}\left\{D E_{x}: x \in G \cap P\right\}
$$

where $\mathrm{P}$ represents the pathway, $\mathrm{G}$ represents the GO BP term of each gene, DE represents the expression value of the DEG, $\frac{G \cap P}{G \cup P}$ is the Jaccard similarity coefficient of $\mathrm{G}$ and $\mathrm{P}$ and

$G \cup P \quad$ is the total DEGs associated with $\mathrm{G}$ and P. iii) Based on the bipartite network, the pathways connected with at least one BP term were selected to construct the pathway network. The formula used for the weight of the edge was as follows:

$$
A_{i j}=\sum_{k=1}^{G} w_{G_{k} P_{i}} \times w_{G_{k} P_{j}} ;
$$

iv) The random walk method (28) was used to calculate the interaction of each pathway pair, and the significant interactions were selected. The transfer matrix of the random walk method was as follows:

$$
T_{i j}=\frac{A_{i j}}{\sum_{p}^{N_{p}} A_{i j}} .
$$

$\mathrm{N}_{p}$ represents the total pathways in the network, $\mathrm{T}_{i j}$ represents the probability of one pathway between $\mathrm{Pi}$ and $\mathrm{Pj}$.

The process was repeated using the bootstrap method (29) steps $1-4$, following which the significant P-value of the interaction was obtained.

\section{Results}

Data preprocessing and screenong of DEGs. A total of 19,944 expression probes were obtained from the GSE33630 
A

Data before normalization

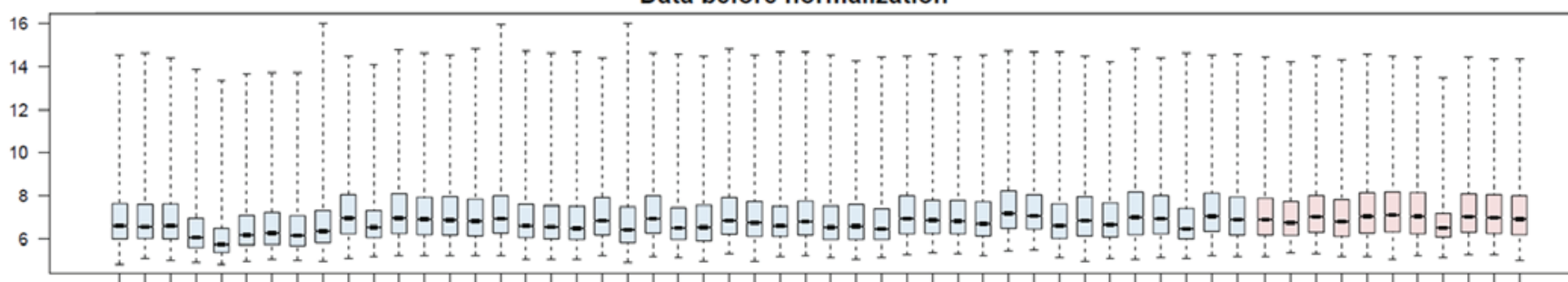

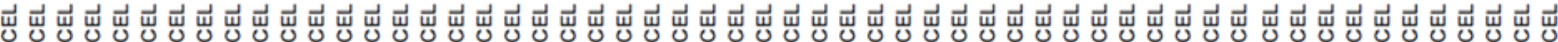

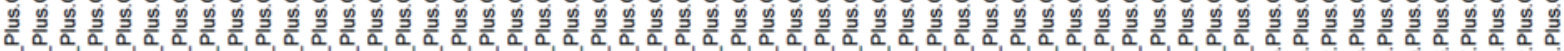

zom

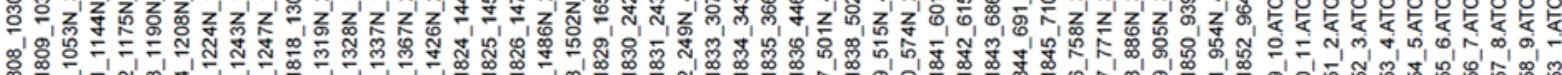

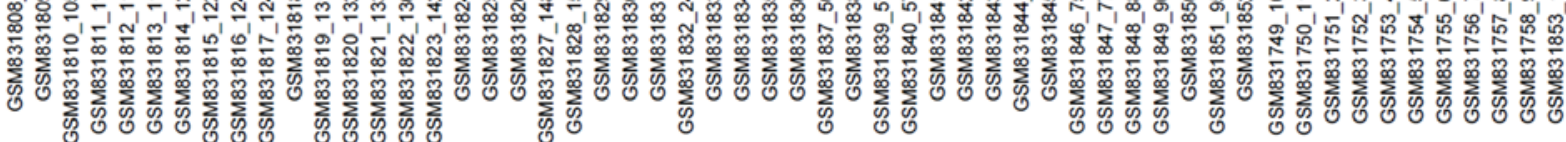

B

Data after normalization

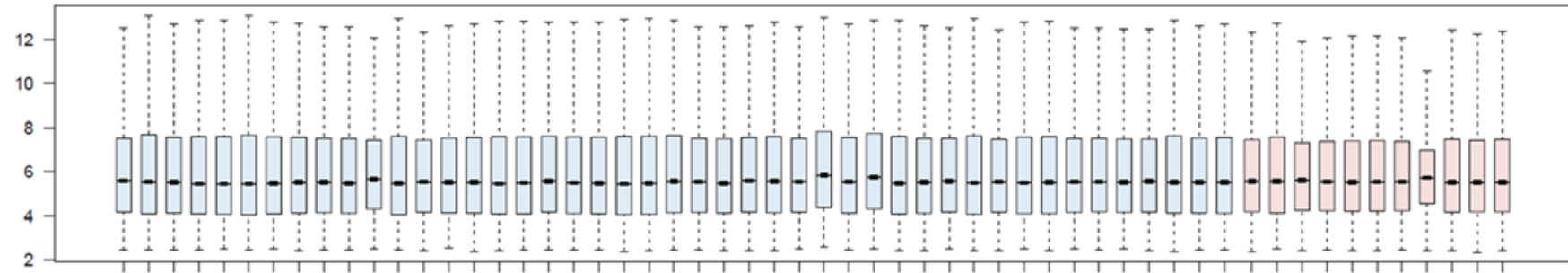

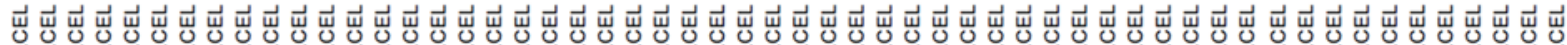

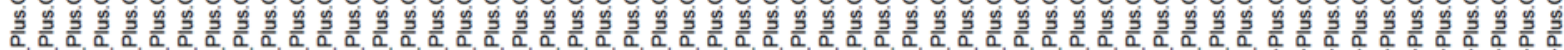

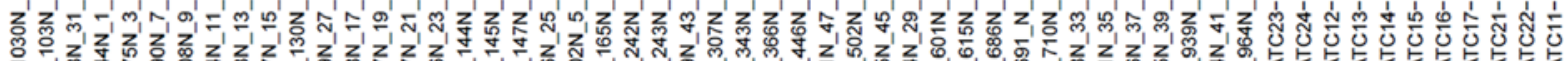

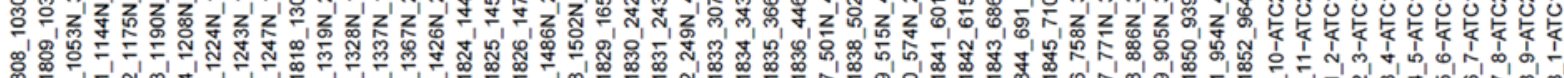

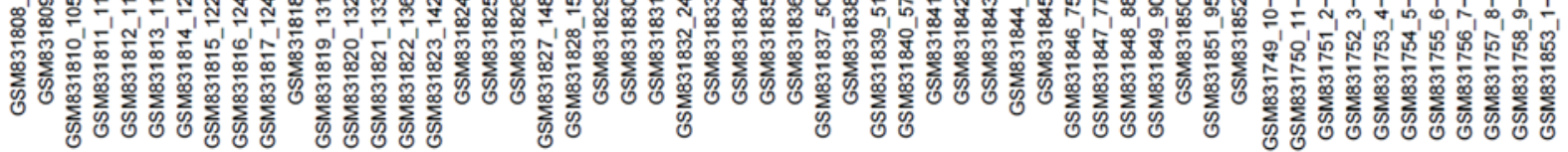

Figure 1. Box figure of gene expression data prior to and following normalization. (A) Data prior to normalization; (B) Data following normalization. The horizontal axis represents the sample and the vertical axis represents the expression value. Black lines indicate the median values. Blue represents the control samples and red represents the anaplastic thyroid carcinoma samples. The vertical axes represent expression signal values.

gene expression profile. The preprocessed data is shown in Fig. 1A and B. The median values indicated that the data were well normalized.

In addition, 665 genes in the ATC samples, which were differentially expressed compared with the NT samples were screened, which comprised 380 upregulated and 285 downregulated DEGs.

GO functions and pathways enrichment analysis of DEGs. The GO terms and pathways of all the selected DEGs were enriched using clusterProfiler with $\mathrm{P}<0.05$ (Table I). The enriched GO terms for the upregulated DEGs in ATC included cellular processes, membrane part and protein binding, whereas single-organism cellular process, extracellular region part and microtubule binding were enriched terms for the downregulated DEGs. In addition, the upregulated DEGs were involved in glycerophospholipid metabolism and metabolic pathways (Table IA), whereas the downregulated DEGs were involved in cytokine-cytokine receptor interaction and the complement and coagulation cascades pathway (Table IB).
PPI interaction network and significant modules. A PPI interaction network of the DEGs was constructed using STRING. A total of four significant modules with the top five node degree were selected (Fig. 2A-D). Functional enrichment analysis for the four modules found that no GO term or pathway was enriched by genes in module D. The enriched GO terms and pathways of the DEGs in these significant modules are shown in Table II.

Interaction analysis between pathways. A total of 406 pathway pairs associated with the DEGs were selected, which were involved in 29 pathways (Fig. 3; Table III). Edges with a high weight indicated a higher level of interaction between two pathways. The interactive pathway pairs with the top five weights are shown in Table IV. hsa04620 interacted with several pathways with high weights, including hsa05323, hsa05142 and hsa05146, indicating hsa04620 was an important pathway. hsa04060 was also identified as an important pathway, which interacted with several other pathways. As shown in Table IV, FBJ murine osteosarcoma viral oncogene homolog (FOS) was the DEG present 
Table I. Top five enriched GO terms and pathways of the DEGs.

ID Term $\quad$ Description $\quad$ Count

A, Top five GO terms and pathways of the upregulated DEGs in ACT

\begin{tabular}{|c|c|c|c|c|}
\hline $\mathrm{BP}$ & GO:0008150 & Biological process & 264 & 4.79E-21 \\
\hline $\mathrm{BP}$ & GO:0044707 & Single-multicellular organism process & 126 & $1.84 \mathrm{E}-06$ \\
\hline $\mathrm{BP}$ & GO:0044699 & Single-organism process & 203 & $1.84 \mathrm{E}-06$ \\
\hline $\mathrm{BP}$ & GO:0009987 & Cellular process & 230 & $1.84 \mathrm{E}-06$ \\
\hline $\mathrm{BP}$ & GO:0032501 & Multicellular organismal process & 127 & $5.99 \mathrm{E}-06$ \\
\hline $\mathrm{CC}$ & GO:0005575 & Cellular component & 284 & $2.26 \mathrm{E}-06$ \\
\hline $\mathrm{CC}$ & GO:0016020 & Membrane & 162 & $9.97 \mathrm{E}-05$ \\
\hline $\mathrm{CC}$ & GO:0044425 & Membrane part & 131 & 0.00042334 \\
\hline $\mathrm{CC}$ & GO:0044459 & Plasma membrane part & 54 & 0.00042334 \\
\hline $\mathrm{CC}$ & GO:0005615 & Extracellular space & 30 & 0.00042334 \\
\hline MF & GO:0003674 & Molecular function & 247 & $9.38 \mathrm{E}-17$ \\
\hline MF & GO:0005515 & Protein binding & 139 & $7.67 \mathrm{E}-05$ \\
\hline MF & GO:0005488 & Binding & 195 & 0.000128045 \\
\hline MF & GO:0016491 & Oxidoreductase activity & 22 & 0.004818428 \\
\hline MF & GO:0008509 & Anion transmembrane transporter activity & 11 & 0.00600053 \\
\hline KEGG & hsa00564 & Glycerophospholipid metabolism & 6 & 0.005474129 \\
\hline KEGG & hsa01100 & Metabolic pathways & 30 & 0.009706045 \\
\hline
\end{tabular}

B, Top five GO terms and pathways of the downregulated DEGs in ACT

\begin{tabular}{lllrl}
\hline BP & GO:0044763 & Single-organism cellular process & 213 & $1.08 \mathrm{E}-26$ \\
BP & GO:0044699 & Single-organism process & 222 & $4.03 \mathrm{E}-26$ \\
BP & GO:0000278 & Mitotic cell cycle & 55 & $7.10 \mathrm{E}-22$ \\
BP & GO:0006950 & Response to stress & 108 & $2.27 \mathrm{E}-21$ \\
BP & GO:0008150 & Biological process & 242 & $5.54 \mathrm{E}-20$ \\
CC & GO:0044421 & Extracellular region part & 69 & $8.67 \mathrm{E}-25$ \\
CC & GO:0005576 & Extracellular region & 88 & $2.08 \mathrm{E}-20$ \\
CC & GO:0031012 & Extracellular matrix & 37 & $2.79 \mathrm{E}-18$ \\
CC & GO:0005615 & Extracellular space & 50 & $1.69 \mathrm{E}-17$ \\
CC & GO:0005578 & Proteinaceous extracellular matrix & 33 & $5.96 \mathrm{E}-17$ \\
MF & GO:0003674 & Molecular function & 234 & $4.95 \mathrm{E}-16$ \\
MF & GO:0005515 & Protein binding & 161 & $5.84 \mathrm{E}-16$ \\
MF & GO:0005488 & Binding & 203 & $4.91 \mathrm{E}-12$ \\
MF & GO:0008017 & Microtubule binding & 16 & $1.28 \mathrm{E}-09$ \\
MF & GO:0015631 & Tubulin binding & 17 & $1.45 \mathrm{E}-08$ \\
KEGG & hsa04512 & Extracellular matrix-receptor interaction & 14 & $7.14 \mathrm{E}-10$ \\
KEGG & hsa05150 & Staphylococcus aureus infection & 8 & $8.10 \mathrm{E}-06$ \\
KEGG & hsa04510 & Focal adhesion & 14 & $1.24 \mathrm{E}-05$ \\
KEGG & hsa04060 & Cytokine-cytokine receptor interaction & 16 & $1.44 \mathrm{E}-05$ \\
KEGG & hsa04610 & Complement and coagulation cascades & $1.56 \mathrm{E}-05$ \\
\hline
\end{tabular}

ACT, anaplastic thyroid carcinoma; DEGs, differentially expressed genes; GO, Gene Ontology; KEGG, Kyoto Encyclopedia of Genes and Genomes; BP, biological process; MF, molecular function; CC, cellular component.

in hsa04620, the Toll-like receptor (TLR) signaling pathway and COL5A1 was the DEG present in hsa04512 (ECM-receptor interaction). Certain cytokines, including CXCL12, CXCL3, interleukin (IL)8, CXCL10 and CCL28, were the genes involved in hsa04060 (cytokine-cytokine receptor interaction pathway).

\section{Discussion}

ATC is an aggressive malignancy of older adults with a low morbidity rate, but a high mortality rate (2). Investigating the mechanism of ATC is beneficial to the treatment and diagnosis 
Table II. Top five enriched GO terms and pathways of differentially expressed genes in the significant modules.

ID Term $\quad$ Description Count P-value

A, Top five enriched GO terms and pathways of DEGs in module A

\begin{tabular}{lllcl}
\hline BP & GO:0007049 & Cell cycle & 57 & $5.95 \mathrm{E}-49$ \\
BP & GO:0000278 & Mitotic cell cycle & 49 & $5.95 \mathrm{E}-49$ \\
BP & GO:0022402 & Cell cycle process & 52 & $2.58 \mathrm{E}-47$ \\
BP & GO:0051301 & Cell division & 36 & $7.19 \mathrm{E}-37$ \\
BP & GO:0000280 & Nuclear division & 32 & $8.81 \mathrm{E}-36$ \\
CC & GO:0005819 & Spindle & 23 & $2.20 \mathrm{E}-26$ \\
CC & GO:0015630 & Microtubule cytoskeleton & 31 & $5.69 \mathrm{E}-23$ \\
CC & GO:0000793 & Condensed chromosome & 18 & $3.83 \mathrm{E}-22$ \\
CC & GO:0000775 & Chromosome, centromeric region & 16 & $8.06 \mathrm{E}-20$ \\
CC & GO:0000777 & Condensed chromosome kinetochore & 13 & $7.98 \mathrm{E}-19$ \\
MF & GO:0005515 & Protein binding & 56 & $6.33 \mathrm{E}-13$ \\
MF & GO:0008017 & Microtubule binding & 12 & $6.33 \mathrm{E}-13$ \\
MF & GO:0015631 & Tubulin binding & 12 & $1.81 \mathrm{E}-11$ \\
MF & GO:0003777 & Microtubule motor activity & 8 & $4.83 \mathrm{E}-10$ \\
MF & GO:0005524 & ATP binding & 23 & $6.79 \mathrm{E}-10$ \\
KEGG & hsa04110 & Cell cycle & 8 & $8.20114825913878 \mathrm{e}-10$ \\
\hline
\end{tabular}

B, Top five enriched GO terms and pathways of DEGs in module B

\begin{tabular}{lllrl}
\hline BP & GO:0006935 & Chemotaxis & 11 & $6.44 \mathrm{E}-14$ \\
BP & GO:0042330 & Taxis & 11 & $6.44 \mathrm{E}-14$ \\
BP & GO:0006954 & Inflammatory response & 9 & $4.30 \mathrm{E}-11$ \\
BP & GO:0040011 & Locomotion & 11 & $1.21 \mathrm{E}-10$ \\
BP & GO:0009605 & Response to external stimulus & 11 & $1.54 \mathrm{E}-10$ \\
CC & GO:0005615 & Extracellular space & 7 & $9.36 \mathrm{E}-06$ \\
CC & GO:0044421 & Extracellular region part & 7 & $3.43 \mathrm{E}-05$ \\
CC & GO:0005576 & Extracellular region & 8 & 0.000169765 \\
CC & GO:0044459 & Plasma membrane part & 6 & 0.004168711 \\
CC & GO:0005886 & Plasma membrane & 8 & 0.012325713 \\
MF & GO:0008009 & Chemokine activity & 7 & $3.55 \mathrm{E}-15$ \\
MF & GO:0042379 & Chemokine receptor binding & 7 & $5.71 \mathrm{E}-15$ \\
MF & GO:0001664 & G-protein coupled receptor binding & 7 & $3.54 \mathrm{E}-11$ \\
MF & GO:0005125 & Cytokine activity & 7 & $4.17 \mathrm{E}-11$ \\
MF & GO:0005126 & Cytokine receptor binding & 7 & $4.20 \mathrm{E}-11$ \\
KEGG & hsa04062 & Chemokine signaling pathway & 8 & $7.14 \mathrm{E}-10$ \\
KEGG & hsa04060 & Cytokine-cytokine receptor interaction & 8 & $8.10 \mathrm{E}-06$ \\
\hline
\end{tabular}

C, Top five enriched GO terms and pathways of DEGs in module C

\begin{tabular}{lllrl}
\hline BP & GO:0030198 & Extracellular matrix organization & 13 & $1.57 \mathrm{E}-16$ \\
BP & GO:0043062 & Extracellular structure organization & 13 & $1.57 \mathrm{E}-16$ \\
BP & GO:0022617 & Extracellular matrix disassembly & 9 & $5.02 \mathrm{E}-15$ \\
BP & GO:0030574 & Collagen catabolic process & 8 & $1.12 \mathrm{E}-13$ \\
BP & GO:0044243 & Multicellular organismal catabolic process & 8 & $1.93 \mathrm{E}-13$ \\
CC & GO:0044421 & Extracellular region part & 18 & $4.69 \mathrm{E}-16$ \\
CC & GO:0031012 & Extracellular matrix & 13 & $8.60 \mathrm{E}-15$ \\
CC & GO:0005581 & Collagen & 9 & $8.60 \mathrm{E}-15$ \\
CC & GO:0005578 & Proteinaceous extracellular matrix & 12 & $4.04 \mathrm{E}-14$ \\
CC & GO:0044420 & Extracellular matrix part & 10 & $4.80 \mathrm{E}-14$
\end{tabular}


Table II. Continued.

\begin{tabular}{|c|c|c|c|c|}
\hline ID & Term & Description & Count & P-value \\
\hline MF & GO:0005515 & Protein binding & 17 & 0.002325685 \\
\hline KEGG & hsa05142 & Chagas disease (American trypanosomiasis) & 7 & $1.8408112078959 \mathrm{e}-08$ \\
\hline
\end{tabular}

DEGs, differentially expressed genes; GO, Gene Ontology; KEGG, Kyoto Encyclopedia of Genes and Genomes; BP, biological process; MF, molecular function; CC, cellular component.

Table III. 29 enriched significant pathways in the latent pathway identification analysis network.

\begin{tabular}{ll}
\hline Path ID & \\
\hline hsa04512 & \multicolumn{1}{c}{ Description } \\
hsa04974 & Protein digestion and absorption \\
hsa04060 & Cytokine-cytokine receptor interaction \\
hsa05150 & Staphylococcus aureus infection \\
hsa04610 & Complement and coagulation cascades \\
hsa05146 & Amoebiasis \\
hsa05323 & Rheumatoid arthritis \\
hsa04380 & Osteoclast differentiation \\
hsa04062 & Chemokine signaling pathway \\
hsa04620 & Toll-like receptor signaling pathway \\
hsa04145 & Phagosome \\
hsa04640 & Hematopoietic cell lineage \\
hsa04510 & Focal adhesion \\
hsa04670 & Leukocyte transendothelial migration \\
hsa05144 & Malaria \\
hsa04350 & Transforming growth factor- $\beta$ signaling \\
pathway \\
hsa05142
\end{tabular}

of ATC. In the present study, the key genes and significant pathways associated with ATC were analyzed using ATC and NT samples from the GSE33630 gene expression profile. Several key genes, including CXCL10, FOS, COL5A1 and IL8, and the TLR signaling pathway, ECM-receptor interaction and cytokine-cytokine receptor interaction pathway, in which these key genes were involved, were identified using LPIA. However, due to the different selection methods and samples, the findings of the present study were different from those of previous studies, which used the same gene expression profile (GSE33630) of thyroid cancer. Hébrant et al (14) reported that a more marked EMT was the qualitative difference between PTC and ATC, by examining 11 ATC and 48 PTC samples in GSE33630. He et al (15) found that the p53 signaling pathway, which included 13 genes, was important for thyroid cancer using gene set enrichment and meta-analysis. Xu et al (16) selected the BCL2, MRPS31, ID4, RASAL2, DLG2, MY01B, ZBTB5, PRKCQ, PPP6C genes and an miscRNA on considering NT, ATC and PTC together. Therefore, further experimental validation is required.

Certain types of cancer are associated with inflammatory or infectious diseases, and inflammatory factors are associated with ATC. CXCL10 is a chemokine, which produces a ligand for the receptor, CXCR3, and has a paralog of CXCL9 (30). Graves' disease is an autoimmune thyroid disorder of thyroid cancer (31). The pathogenesis of autoimmune thyroiditis and Graves' disease have been associated with the secretion of CXCL10 stimulated by T helper 1 lymphocytes (32). In addition, Antonelli et al (33) confirmed that human ATC cells produce CXCL10 under the effect of certain cytokines. Thus, autoimmune thyroiditis may contribute to the progression of ATC via the secretion of CXCL10. By contrast, the TLR signaling pathway may act as the novel target for the therapy of inflammatory-associated disease (34), however, the role of the TLR signaling pathway in ATC remains to be fully elucidated. Mardente et al (35) reported that the late inflammatory cytokine, high-mobility group box 1 , can signal danger to the immune system via the TLR in PTC cell growth. The serum levels of CXCL10 are high in patients with Graves' disease, and CXCL10 and CXCL9 are important in the recruitment of thyroid cells (36). The data obtained in the present study showed that the TLR signaling pathway interacted with the cytokine-cytokine pathway, and the downregulated expression of CXCL10 was involved in the two pathways. This indicated that these two pathways were important in the progression of ATC and that CXCL10 may act as a therapeutic target of ATC.

The present study also showed that the downregulation of IL- 8 was important in module B and in the TLR signaling pathway, suggesting it may be important to ATC. IL-8 is a CXC chemokine, which has the antimicrobial functions, and can activate the chemotaxis of neutrophils, T lymphocytes and eosnophils (37). Several cytokines have been found to be associated with thyroid cancer. The transfection of IL-12 in the thyroid can potentially inhibit tumor 

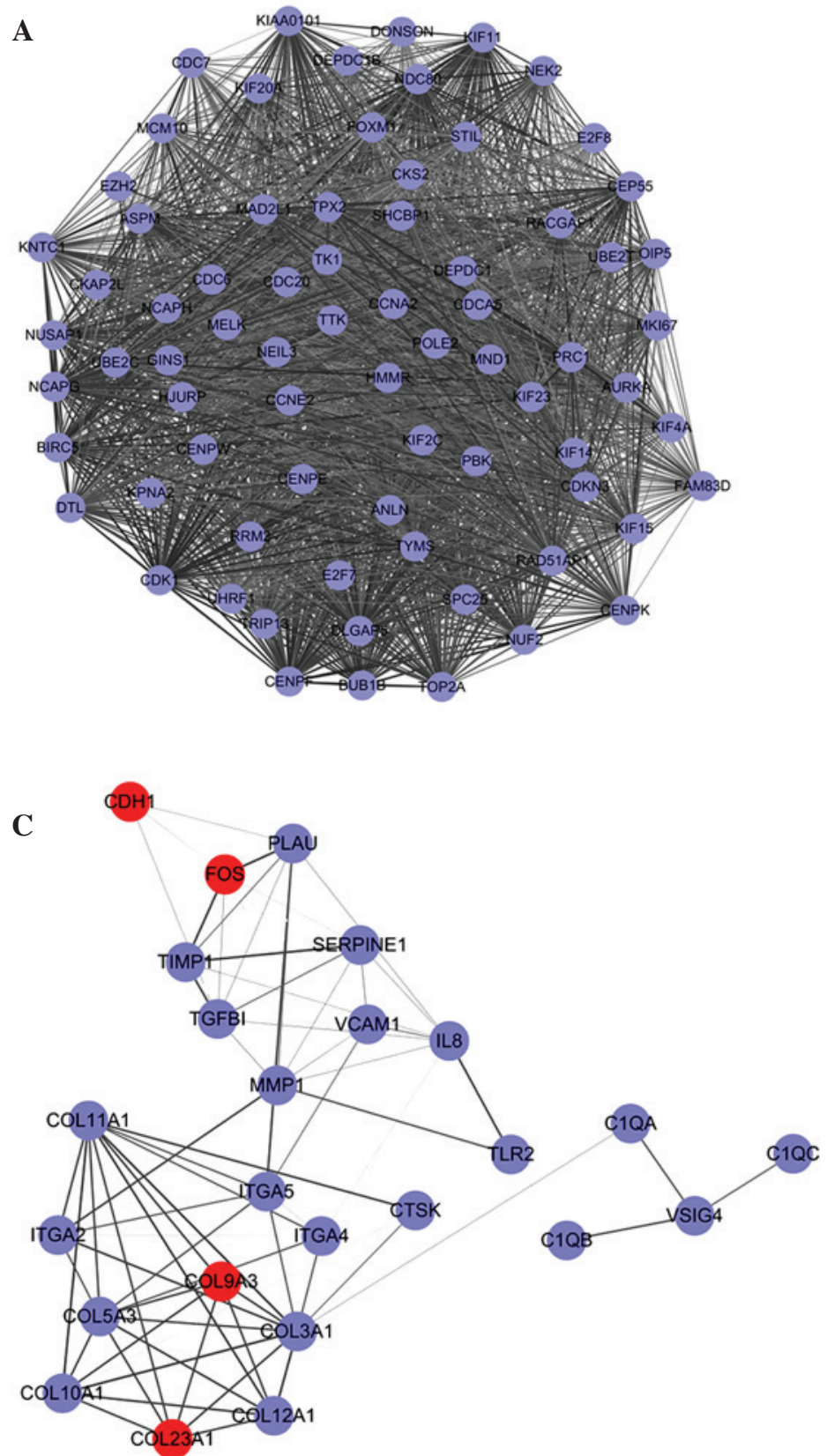

B

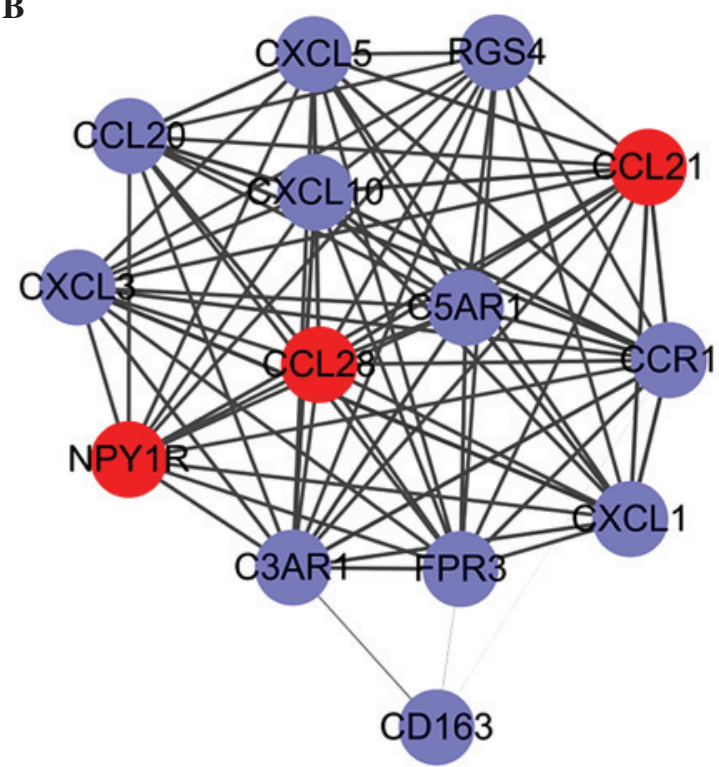

D

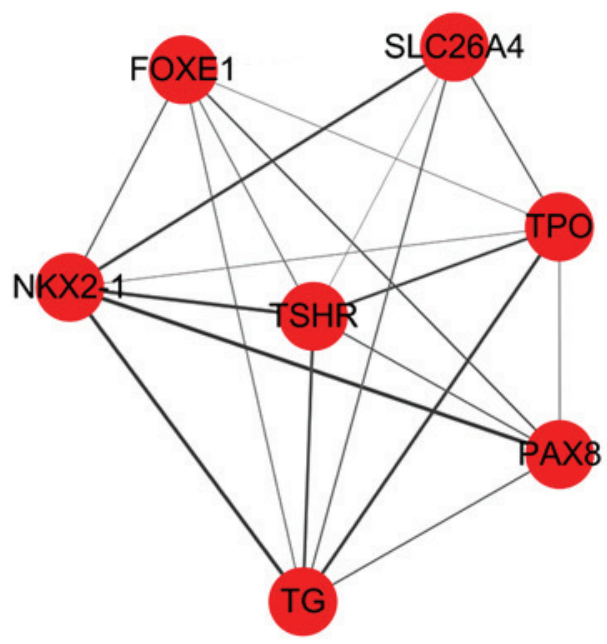

Figure 2. Modules containing the top five node degrees in the protein-protein interaction network. Red indicates upregulated DEGs, purple indicates downregulated DEGs. Modules A-D are the four significant modules. DEGs, differentially expressed genes. Lines between DEGs represent interactions between them.

growth and may be a potential tumor vaccine (38). In a study by Kobawala et al (39) the serum levels of IL-8 in patients with ATC were higher, compared with those in healthy individuals, and it may be a target for the diagnosis and therapeutic treatment of ATC. The findings of the present study indicated that the downregulation of IL- 8 assisted in the evasion of immune surveillance, leading to ATC. Therefore, IL- 8 may be a biomarker for the diagnosis of ATC.

FOS is a member of the FOS family, which acts as a regulator of cell proliferation, differentiation and apoptotic cell death (40). The role of FOS in ATC remains to be fully elucidated, however, studies have demonstrated that c-FOS can bind to the promoter, cellular FLICE-inhibitory protein, to function in prostate cancer cells (41). c-FOS signaling is regulated by protein kinase $\mathrm{R}$ in the promotion of cell proliferation in hepatocellular cancer (42). The cell proliferation instability-related gene cluster is an important factor in ATC transformation (43). Thus, the FOS protein may be crucial in ATC formation. In the present study, the upregulation of FOS was present in the TLR signaling pathway, suggesting that FOS may be important in contributing to ATC formation via the TLR signaling pathway.

ECM is a protein, which is crucial in thyroid tumor invasion, metastasis and diagnosis (44). COL, a low abundance fibrillar collagen of the COL family, is a component of ECM (45). The activity of base membrane COL4 indicates the cell proliferation and metastatic abilities of thyroid cancer (46). Kusunoki et al (47) showed that the level of COL4 collagenase in patients with thyroid cancer was higher, compared with that in the normal thyroid, and COL4 may assist in predicting 
Table IV. Interacting pairs of pathways with top 10 weights.

\begin{tabular}{|c|c|c|c|}
\hline Pathway 1 and description & Pathway 2 and description & Weight & Co-genes \\
\hline hsa05323: & hsa04620: & 0.492651941 & $\mathrm{CTSK}^{\mathrm{a}} / \mathrm{CTSO}^{\mathrm{a}} / \mathrm{FOS} / \mathrm{IL}^{\mathrm{a}} / \mathrm{TLR}^{\mathrm{a}}$ \\
\hline Rheumatoid arthritis & Toll-like receptor signaling pathway & & \\
\hline hsa05142: & hsa04620: & 0.464920784 & IL8 8 /TLR2 $/$ FOS \\
\hline $\begin{array}{l}\text { Chagas disease (American } \\
\text { trypanosomiasis) }\end{array}$ & Toll-like receptor signaling pathway & & \\
\hline hsa05146: & hsa04620: & 0.37284676 & IL8 ${ }^{\mathrm{a}} / \mathrm{TLR} 2^{\mathrm{a}} / \mathrm{CD} 14^{\mathrm{a}}$ \\
\hline Amoebiasis & Toll-like receptor signaling pathway & & \\
\hline hsa04620 & hsa04060: & 0.351558647 & IL8 $^{\mathrm{a}} / \mathrm{CXCL} 10^{\mathrm{a}}$ \\
\hline $\begin{array}{l}\text { Toll-like receptor signaling } \\
\text { pathway }\end{array}$ & Cytokine-cytokine receptor interaction & & \\
\hline hsa04062: & hsa04060: & 0.33933744 & $\mathrm{CCR}^{\mathrm{a}} / \mathrm{CXCL}^{\mathrm{a}} / \mathrm{CXCL}^{\mathrm{a}} / \mathrm{IL}^{\mathrm{a}} /$ \\
\hline Chemokine signaling pathway & Cytokine-cytokine receptor interaction & & $\begin{array}{l}\text { CXCL10 } 1 \text { CCL28/CCL8 }{ }^{\mathrm{a}} / \\
\mathrm{CCL} 13^{\mathrm{a}} / \mathrm{CCL} 18^{\mathrm{a}} / \mathrm{CCL} 21 / \mathrm{CXCL}^{\mathrm{a}}\end{array}$ \\
\hline hsa04380: & hsa04620: & 0.327230722 & $\mathrm{CTSK}^{\mathrm{a}} / \mathrm{FOS}$ \\
\hline Osteoclast differentiation & Toll-like receptor signaling pathway & & \\
\hline hsa04974: & hsa04512: & 0.31737287 & COL5A1\% COL6A33a/COL11A1ª \\
\hline Protein digestion and absorption & ECM-receptor interaction & & COL5A3 ${ }^{\mathrm{a}}$ \\
\hline hsa04512: & hsa04510: & 0.307854633 & COL5A1ªLOA33/COL11A1\% \\
\hline ECM-receptor interaction & Focal adhesion & & 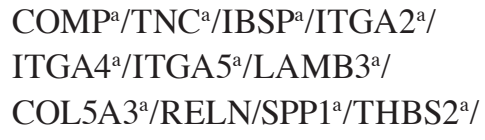 \\
\hline hsa04145: & hsa04620: & 0.305699295 & $\mathrm{TLR}^{\mathrm{a}} / \mathrm{CD} 14^{\mathrm{a}}$ \\
\hline Phagosome & Toll-like receptor signaling pathway & & \\
\hline hsa05150: & hsa04610: & 0.272281647 & $\mathrm{CFD} / \mathrm{C} 1 \mathrm{QA}^{\mathrm{a}} / \mathrm{C} 1 \mathrm{QB}{ }^{\mathrm{a}} / \mathrm{C} 1 \mathrm{Q} \mathrm{C}^{\mathrm{a}} /$ \\
\hline Staphylococcus aureus infection & Complement and coagulation cascades & & C3AR1 $1^{\mathrm{a}} / \mathrm{C} 5 \mathrm{AR} 1^{\mathrm{a}}$ \\
\hline
\end{tabular}

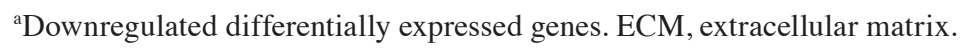

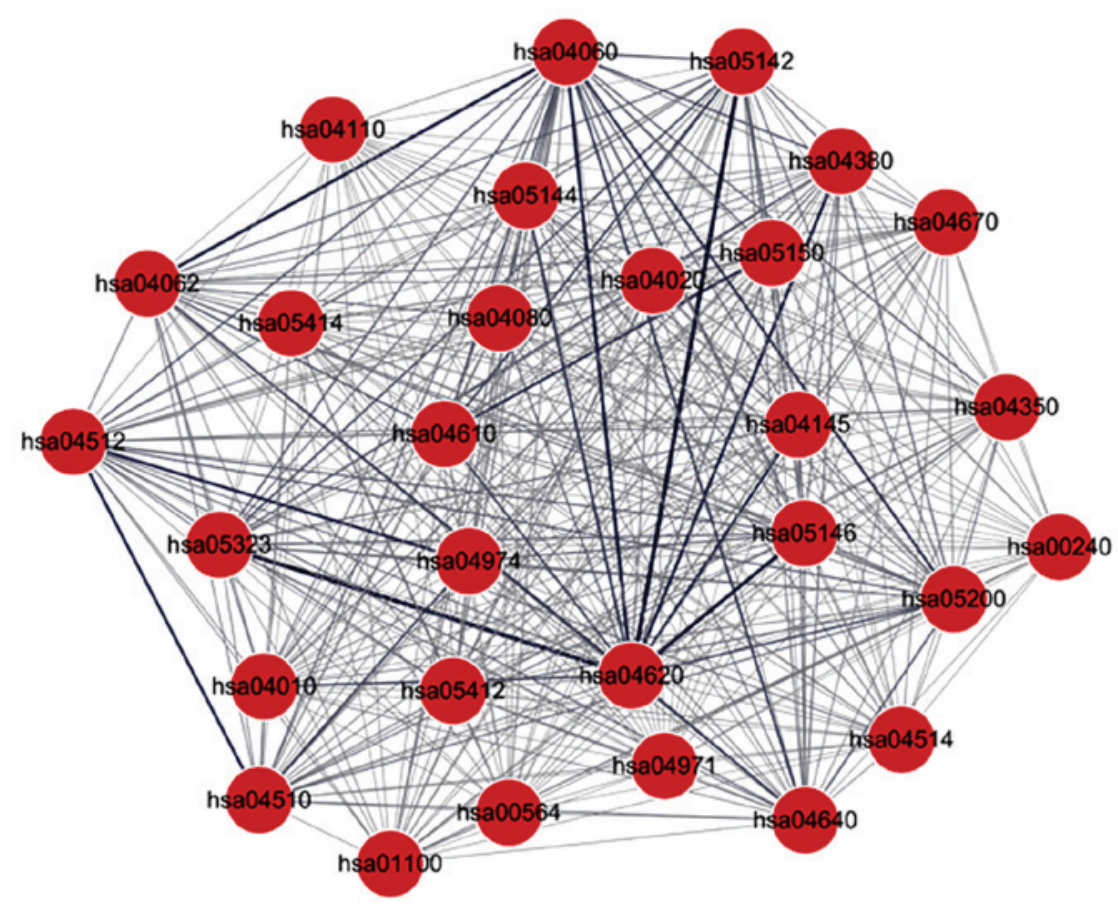

Figure 3. Interaction network of the pathways involved with the differentially expressed genes. Lines between DEGs represent interactions between them. 
the invasion and metastasis of thyroid cancer. The expression of COL5A1 is suppressed by microRNA-145 in anaplastic meningionas (48). Downregulated COL5A1 can decrease the adhesion of tumor cells via the ECM-receptor interaction pathway, enhancing tumor metastasis (49). COL11A1 and COL5A1 promote cell migration and tumor progression in mice (50). In the present study, the findings showed that COL5A1 and COL11A1 were downregulated and were present in the ECM-receptor interaction pathway, suggesting that the two genes may be important in the progression of ATC via the ECM-receptor interaction pathway.

In conclusion, the present study analyzed the key genes and significant pathways in ATC, compared with NT samples. The TLR signaling pathway, ECM-receptor interaction and cytokine-cytokine receptor interaction pathway were found to be closely associated with ATC. CXCL10 may be of benefit in ATC treatment, FOS may be involved in ATC formation, COL5A1 and COL11A1 may promote the progression of ATC, and IL8 may act as a biomarker for the diagnosis of ATC. The results of the present study provide a basis for further clinical molecular target therapy experiments for ATC.

\section{Acknowledgements}

This study was supported by the National Natural Science Foundation of China (grant no. 81372860).

\section{References}

1. Siegel R, Naishadham D and Jemal A: Cancer statistics, 2013. CA Cancer J Clin 63: 11-30, 2013.

2. Neff RL, Farrar WB, Kloos RT and Burman KD: Anaplastic thyroid cancer. Endocrinol Metab Clin North Am 37: 525-538, 2008.

3. Smallridge RC, Marlow LA and Copland JA: Anaplastic thyroid cancer: Molecular pathogenesis and emerging therapies. Endocr Relat Cancer 16: 17-44, 2009.

4. Are C and Shaha AR: Anaplastic thyroid carcinoma: Biology, pathogenesis, prognostic factors, and treatment approaches. Ann Surg Oncol 13: 453-464, 2006.

5. Passaro C, Abagnale A, Libertini S, Volpe M, Botta G, Cella L, Pacelli R, Halldèn G, Gillespie D and Portella G: Ionizing radiation enhances d1922-947-mediated cell death of anaplastic thyroid carcinoma cells. Endocr Relat Cancer 20: 633-647, 2013

6. Harach HR, Galíndez M, Campero M and Ceballos GA: Undifferentiated (anaplastic) thyroid carcinoma and iodine intake in Salta, Argentina. Endocr Pathol 24: 125-131, 2013.

7. Marlow LA, Von Roemeling CA, Cooper SJ, Zhang Y, Rohl SD, Arora S, Gonzales IM, Azorsa DO, Reddi HV, Tun HW, et al: Foxo3a drives proliferation in anaplastic thyroid carcinoma through transcriptional regulation of cyclin A1: A paradigm shift that impacts current therapeutic strategies. J Cell Sci 125: 4253-4263, 2012.

8. Kim SH, Kang JG, Kim CS, Ihm SH, Choi MG, Yoo HJ and Lee SJ: CCAAT/enhancer-binding protein-homologous protein sensitizes to SU5416 by modulating p21 and PI3K/Akt signal pathway in FRO anaplastic thyroid carcinoma cells. Horm Metab Res 45: 9-14, 2013.

9. $\mathrm{Yu}$ XM, Jaskula-Sztul R, Ahmed K, Harrison AD, Kunnimalaiyaan $\mathrm{M}$ and Chen $\mathrm{H}$ : Resveratrol induces differentiation markers expression in anaplastic thyroid carcinoma via activation of Notch1 signaling and suppresses cell growth. Mol Cancer Ther 12: 1276-1287, 2013.

10. Lim YC and Cha YY: Epigallocatechin-3-gallate induces growth inhibition and apoptosis of human anaplastic thyroid carcinoma cells through suppression of EGFR/ERK pathway and cyclin B1/CDK1 complex. J Surg Oncol 104: 776-780, 2011.

11. Becker N, Chernock RD, Nussenbaum B and Lewis JS Jr: Prognostic significance of $\beta$-human chorionic gonadotropin and PAX8 expression in anaplastic thyroid carcinoma. Thyroid 24: 319-326, 2014.
12. Kim KS, Min J-K, Liang ZL, Lee K, Lee JU, Bae KH, Lee MH, Lee SE, Ryu MJ, Kim SJ, et al: Aberrant 11 cell adhesion molecule affects tumor behavior and chemosensitivity in anaplastic thyroid carcinoma. Clin Cancer Res 18: 3071-3078, 2012.

13. Gauchotte G, Philippe C, Lacomme S, Léotard B, Wissler MP, Allou L, Toussaint B, Klein M, Vignaud JM and Bressenot A: BRAF, p53 and SOX2 in anaplastic thyroid carcinoma: Evidence for multistep carcinogenesis. Pathology 43: 447-452, 2011.

14. Hébrant A, Dom G, Dewaele M, Andry G, Trésallet C, Leteurtre E, Dumont JE and Maenhaut C: mRNA expression in papillary and anaplastic thyroid carcinoma: Molecular anatomy of a killing switch. PLoS One 7: e37807, 2012.

15. He W, Qi B, Zhou Q, Lu C, Huang Q, Xian L and Chen M: Key genes and pathways in thyroid cancer based on gene set enrichment analysis. Oncol Rep 30: 1391-1397, 2013.

16. Xu Y, Deng Y, Ji Z, Liu H, Liu Y, Peng H, Wu J and Fan J: Identification of thyroid carcinoma related genes with mRMR and shortest path approaches. PLoS One 9: e94022, 2014.

17. Gautier L, Cope L, Bolstad BM and Irizarry RA: affy-analysis of Affymetrix GeneChip data at the probe level. Bioinformatics 20: 307-315, 2004.

18. Gentleman RC, Carey VJ, Bates DM, Bolstad B, Dettling M, Dudoit S, Ellis B, Gautier L, Ge Y, Gentry J, et al: Bioconductor: Open software development for computational biology and bioinformatics. Genome Biol 5: R80, 2004.

19. Smyth GK: Limma: Linear models for microarray data, in Bioinformatics and Computational Biology Solutions using $\mathrm{R}$ and Bioconductor. Gentleman R, Carey VJ, Huber W, Irizarry RA and Dudoit S (eds). Springer, New Yotk, NY, pp397-420, 2005.

20. Hulsegge I, Kommadath A and Smits MA: Globaltest and GOEAST: Two different approaches for gene ontology analysis. BMC Proc 3 (Suppl 4): S10, 2009.

21. Kanehisa M and Goto S: KEGG: Kyoto encyclopedia of genes and genomes. Nucleic Acids Res 28: 27-30, 2000.

22. Huang da W and Sherman BT and Lempicki RA: Systematic and integrative analysis of large gene lists using DAVID bioinformatics resources. Nat Protoc 4: 44-57, 2009.

23. Franceschini A, Szklarczyk D, Frankild S, Kuhn M, Simonovic M, Roth A, Lin J, Minguez P, Bork P, von Mering C and Jensen LJ: STRING v9. 1: Protein-protein interaction networks, with increased coverage and integration. Nucleic Acids Res 41 (Database issue): D808-D815, 2013.

24. Kohl M, Wiese S and Warscheid B: Cytoscape: Software for visualization and analysis of biological networks. Methods Mol Biol 696: 291-303, 2011

25. Niissalo A: Cytoscape and its Plugins. Department of Computer Science University of Helsinki Finland, 2007.

26. Pham L, Christadore L, Schaus S and Kolaczyk ED: Network-based prediction for sources of transcriptional dysregulation using latent pathway identification analysis. Proc Natl Acad Sci USA 108: 13347-13352, 2011.

27. Yu G, Wang LG, Han Y and He QY: clusterProfiler: An R package for comparing biological themes among gene clusters. OMICS 16: 284-287, 2012.

28. Jamali $\mathbf{M}$ and Ester M: TrustWalker: A random walk model for combining trust-based and item-based recommendation. In Proceedings of the 15th ACM SIGKDD International Conference on Knowledge Discovery and Data Mining. ACM, New York, NY, pp397-406. 2009.

29. Kim JH: Boostrap prediction intervals and bias-corrected forecasting. http://cran.rproject.org/web/packages/BootPR/index.html. 2009.

30. Ruffilli I, Ferrari SM, Colaci M, Ferri C, Politti U, Antonelli A and Fallahi P: CXCR3 and CXCL10 in autoimmune thyroiditis. Clin Ter 165: e237-e242, 2014 (In Italian).

31. Baloch ZW, Livolsi VA, Asa SL, Rosai J, Merino MJ, Randolph G, Vielh P, DeMay RM, Sidawy MK and Frable WJ: Diagnostic terminology and morphologic criteria for cytologic diagnosis of thyroid lesions: A synopsis of the national cancer institute thyroid fine-needle aspiration state of the science conference. Diagn Cytopathol 36: 425-437, 2008.

32. Fallahi P, Ferrari SM, Corrado A, Giuggioli D, Ferri C and Antonelli A: Targeting chemochine (C-X-C motif) receptor 3 in thyroid autoimmunity. Recent Pat Endocr Metab Immune Drug Discov 8: 95-101, 2014.

33. Antonelli A,Ferrari SM,Fallahi P,Piaggi S,DiDomenicantonio A, Galleri D, Santarpia L, Basolo F, Ferrannini E and Miccoli P: Variable modulation by cytokines and thiazolidinediones of the prototype Th1 chemokine CXCL10 in anaplastic thyroid cancer. Cytokine 59: 218-222, 2012. 
34. Lin Q, Li M, Fang D, Fang J and Su SB: The essential roles of Toll-like receptor signaling pathways in sterile inflammatory diseases. Int Immunopharmacol 11: 1422-1432, 2011.

35. Mardente S, Mari E, Consorti F, Di Gioia C, Negri R, Etna M, Zicari A and Antonaci A: HMGB1 induces the overexpression of miR-222 and miR-221 and increases growth and motility in papillary thyroid cancer cells. Oncol Rep 28: 2285-2289, 2012.

36. Romagnani P, Rotondi M, Lazzeri E, Lasagni L, Francalanci M, Buonamano A, Milani S, Vitti P, Chiovato L, Tonacchera M, et al: Expression of IP-10/CXCL10 and MIG/CXCL9 in the thyroid and increased levels of IP-10/CXCL10 in the serum of patients with recent-onset Graves' disease. Am J Pathol 161: 195-206, 2002.

37. Yousefi S, Hemmann S, Weber M, Hölzer C, Hartung K, Blaser K and Simon HU: IL-8 is expressed by human peripheral blood eosinophils. Evidence for increased secretion in asthma. J Immunol 154: 5481-5490, 1995.

38. Tanaka K, Towata S, Nakao K, Mizuguchi H, Hayakawa T, Niwa M, Ishii N and Nagayama Y: Thyroid cancer immuno-therapy with retroviral and adenoviral vectors expressing granulocyte macrophage colony stimulating factor and interleukin-12 in a rat model. Clin Endocrinol 59: 734-742, 2003.

39. Kobawala TP, Patel GH, Gajjar DR, Patel KN, Thakor PB, Parekh UB, Patel KM, Shukla SN and Shah PM: Clinical utility of serum interleukin- 8 and interferon-alpha in thyroid diseases. J Thyroid Res 2011: 270149, 2011.

40. Bazhenova Elu, Kulikov AV, Tikhonova MA, Tsybko AS and Popova NK: Effects of stress on corticosterone level, expression of c-Fos gene and serotonin turnover in brain in mice with genetic predisposition to catalepsy. Ross Fiziol Zh Im I M Sechenova 98: 1070-1078, 2012 (In Russian).

41. Zhang X, Zhang L, Yang H, Huang X, Otu H, Libermann TA, DeWolf WC, Khosravi-Far R and Olumi AF: c-Fos as a proapoptotic agent in TRAIL-induced apoptosis in prostate cancer cells. Cancer Res 67: 9425-9434, 2007.
42. Watanabe T, Hiasa Y, Tokumoto Y, Hirooka M, Abe M, Ikeda Y, Matsuura B, Chung RT and Onji M: Protein kinase R modulates c-Fos and c-Jun signaling to promote proliferation of hepatocellular carcinoma with hepatitis C virus infection. PLoS One 8: e67750, 2013.

43. Salvatore G, Nappi TC, Salerno P, Jiang Y, Garbi C, Ugolini C, Miccoli P, Basolo F, Castellone MD, Cirafici AM, et al: A cell proliferation and chromosomal instability signature in anaplastic thyroid carcinoma. Cancer Res 67: 10148-10158, 2007.

44. Ingeson-Carlsson C and Nilsson M: Dual contribution of MAPK and PI3K in epidermal growth factor-induced destabilization of thyroid follicular integrity and invasion of cells into extracellular matrix. Exp Cell Res 326: 210-218, 2014.

45. Brown JC, Miller CJ, Posthumus M, Schwellnus MP and Collins M: The COL5A1 gene, ultra-marathon running performance, and range of motion. Int J Sports Physiol Perform 6: 485-496, 2011

46. Kusunoki T, Nishida S, Kimoto-Kinoshita S, Murata K, Satou T and Tomura T: Type IV collagenase and immunostaining of type IV collagen in human thyroid tumors. Auris Nasus Larynx 27: 161-165, 2000.

47. Kusunoki T, Nishida S, Kimoto-Kinoshita S, Murata K, Satou T and Tomura T: Type IV collagen, type IV collagenase activity and ability of cell proliferation in human thyroid tumours. Asian J Surg 25: 304-308, 2002

48. Kliese N, Gobrecht P, Pachow D, Andrae N, Wilisch-Neumann A, Kirches E, Riek-Burchardt M, Angenstein F, Reifenberger G, Riemenschneider MJ, et al: miRNA-145 is downregulated in atypical and anaplastic meningiomas and negatively regulates motility and proliferation of meningioma cells. Oncogene 32: 4712-4720, 2013.

49. Christner PJ and Ayitey S: Extracellular matrix containing mutated fibrillin-1 (Fbn1) down regulates Colla1, Colla2, Col3a1, Col5a1, and Col5a2 mRNA levels in Tsk/+ and Tsk/Tsk embryonic fibroblasts. Amino Acids 30: 445-451, 2006.

50. Cheon DJ, Tong Y, Sim MS, Dering J,Berel D, Cui X, Lester J, Beach JA, Tighiouart M, Walts AE, et al: A collagen-remodeling gene signature regulated by TGF- $\beta$ signaling is associated with metastasis and poor survival in serous ovarian cancer. Clin Cancer Res 20: 711-723, 2014. 